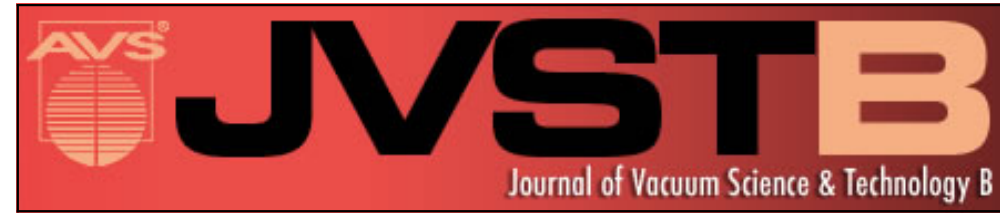

\title{
Effects of photowashing treatment on electrical properties of an AIGaN/GaN heterostructure field-effect transistor
}

Kyoung Jin Choi, Chang Min Jeon, Ho Won Jang, and Jong-Lam Lee

Citation: Journal of Vacuum Science \& Technology B 20, 1574 (2002); doi: 10.1116/1.1491554

View online: http://dx.doi.org/10.1116/1.1491554

View Table of Contents: http://scitation.aip.org/content/avs/journal/jvstb/20/4?ver=pdfcov

Published by the AVS: Science \& Technology of Materials, Interfaces, and Processing

\section{Articles you may be interested in}

Thermal distributions of surface states causing the current collapse in unpassivated AIGaN/GaN heterostructure field-effect transistors

Appl. Phys. Lett. 86, 012106 (2005); 10.1063/1.1844610

Localized variations in electronic structure of AIGaN/GaN heterostructures grown by molecular-beam epitaxy Appl. Phys. Lett. 79, 2749 (2001); 10.1063/1.1410342

Chemistry and electrical properties of surfaces of $\mathrm{GaN}$ and GaN/AIGaN heterostructures

J. Vac. Sci. Technol. B 19, 1675 (2001); 10.1116/1.1383078

Polarization effects, surface states, and the source of electrons in AIGaN/GaN heterostructure field effect transistors

Appl. Phys. Lett. 77, 250 (2000); 10.1063/1.126940

Trap characterization by gate-drain conductance and capacitance dispersion studies of an AlGaN/GaN heterostructure field-effect transistor

J. Appl. Phys. 87, 8070 (2000); 10.1063/1.373499

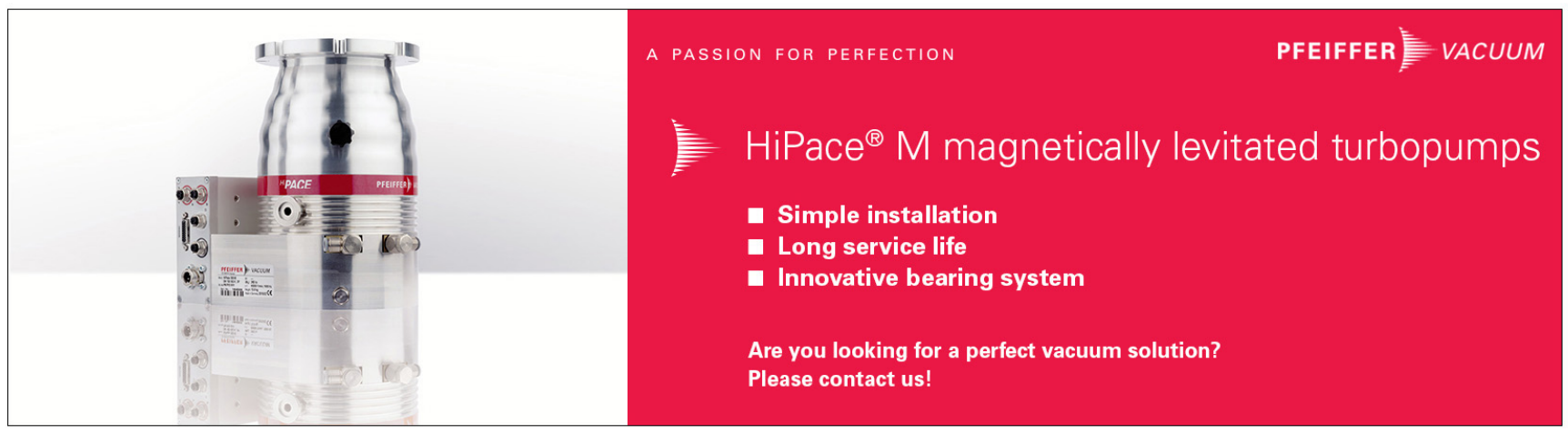




\title{
Effects of photowashing treatment on electrical properties of an AlGaN/GaN heterostructure field-effect transistor
}

\author{
Kyoung Jin Choi, Chang Min Jeon, Ho Won Jang, and Jong-Lam Lee ${ }^{\text {a) }}$ \\ Department of Materials Science and Engineering, Pohang University of Science and Technology, Pohang, \\ Kyungbuk 790-784, Korea
}

(Received 6 March 2002; accepted 13 May 2002)

\begin{abstract}
The effects of photowashing treatment on the electrical properties of an $\mathrm{AlGaN} / \mathrm{GaN}$ heterostructure field-effect transistor were studied by observing changes in atomic composition and band bending at the surface of AlGaN through synchrotron radiation photoemission spectroscopy. The surface treatment produced group-III oxides on the surface of AlGaN, leaving $\mathrm{N}$ vacancies behind. Both the gate leakage current $\left(I_{G D}\right)$ and drain current $\left(I_{D S}\right)$ simultaneously decreased after the treatment. The decrease of $I_{G D}$ was due to a delay in movement of the electrons, namely, trapping and detrapping. The trapped electrons reduced the effective channel thickness, and led to the reduction of $I_{D S}$. (c) 2002 American Vacuum Society. [DOI: 10.1116/1.1491554]
\end{abstract}

AlGaN/GaN heterostructures have made III nitrides suitable for high power and high temperature transistors. The excellent microwave performance of AlGaN/GaN heterostructure field-effect transistors (HFETs) was demonstrated. ${ }^{1,2}$ Because of the inherent chemical stability of $\mathrm{GaN}$, device fabrication processes that involve the AlGaN/ GaN materials system have usually relied on dry etching which is known to damage surfaces and introduce charge trapping centers on etched surfaces. ${ }^{3}$ Surface damage induced during a recessed gate process in the fabrication of HFETs especially could drastically reduce the gate breakdown voltage unless very low plasma power is used. ${ }^{4}$

Ultraviolet (UV)-assisted wet etching of group-III nitrides, such as $\mathrm{GaN}$ and $\mathrm{AlGaN}$, was actively studied due to the low amount of physical damage introduced onto etched surfaces in the gate recess etching process. ${ }^{5,6}$ Previously, the photoluminescence intensity on the surface of GaAs sharply increased after photowashing treatment. ${ }^{7}$ This was explained by a reduction in the surface state density that causes electron-hole recombination. However, exposing the surface of group-III nitrides to UV illumination could change materials properties such as the concentration of surface states and the Fermi level position at the group-III nitride surface because of different photon-induced chemical reaction at surfaces of GaAs and group-III nitrides. Until now, no results were, however, reported on the type of point defect related to such surface states and to resultant changes in the electrical properties of HFETs.

In this work, the changes in atomic composition and surface states at the surface of AlGaN caused by photowashing treatment were investigated by synchrotron radiation photoemission spectroscopy (SRPES). The effect of surface treatment on the electrical properties of $\mathrm{AlGaN} / \mathrm{GaN}$ HFETs was examined by both current-voltage $(I-V)$ and transconductance $\left(G_{m}\right)$ dispersion measurements. From these, the origin of changes in electrical properties caused by the photowashing treatment was proposed.

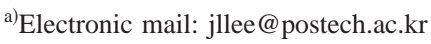

The undoped AlGaN/GaN heterostructure used in this work was grown by metalorganic chemical vapor deposition (MOCVD) on a sapphire substrate. An undoped GaN buffer layer with a thickness of $1.2 \mu \mathrm{m}$ was grown, followed by the growth of 270 - $\AA$-thick undoped $\mathrm{Al}_{0.25} \mathrm{Ga}_{0.75} \mathrm{~N}$. The electron mobility and sheet concentration were respectively, $510 \mathrm{~cm}^{2} / \mathrm{V} \mathrm{s}$ and $3 \times 10^{13} / \mathrm{cm}^{3}$, determined by Hall measurements.

In the fabrication of the $\mathrm{AlGaN} / \mathrm{GaN}$ HFET, an active region was defined using $\mathrm{Cl}_{2}$ inductively coupled plasma. $\mathrm{Ti} / \mathrm{Al}$ ohmic contacts were deposited by an electron beam evaporator, followed by rapid thermal annealing at $700{ }^{\circ} \mathrm{C}$ for $1 \mathrm{~min}$. A $1.0 \mu \mathrm{m}$ gate pattern was formed with image reversal lithography and a $0.1-\mu$ m-thick $\mathrm{Pt}$ gate was then deposited on top of the undoped AlGaN layer.

The device fabricated was photowashed by dipping it into de-ionized water under an UV illumination. An unfiltered $\mathrm{Hg}$ arc lamp with an intensity of $10 \mathrm{~mW} / \mathrm{cm}^{2}$ provided uniform illumination over the entire surface of the device. The chemical composition at the surface of AlGaN was characterized using SRPES. In order to obtain depth information on the atomic composition, the take-off angle $\theta$, defined as the angle of photoelectron emission to the surface, was changed from $10^{\circ}$ to $90^{\circ}$.

Figure 1(a) shows the change in gate-to-drain leakage current $\left(I_{G D}\right)$ with the photowashing treatment. The measurement was carried out with the source floated, that is, a twoterminal condition. $I_{G D}$ decreased significantly after being photowashed for $10 \mathrm{~min}$. The drain current $\left(I_{D S}\right)$ also decreased, as shown in Fig. 1(b), taking into consideration the shift of pinch-off voltage $\left(V_{p}\right)$ towards higher gate voltage $\left(V_{G S}\right)$. These results show that surface states that cause the change in electrical properties were produced during the photowashing treatment.

Figure 2 shows the change in $G_{m}$ dispersion of the HFET with the treatment. The values were normalized to the value measured at $1 \mathrm{~Hz}$. The gate and drain biases used were -2.0 and $6.0 \mathrm{~V}$, respectively. Before the treatment, $G_{m}$ was nearly independent of the frequency, but it decreased after the treatment, namely, negative $G_{m}$ dispersion. 

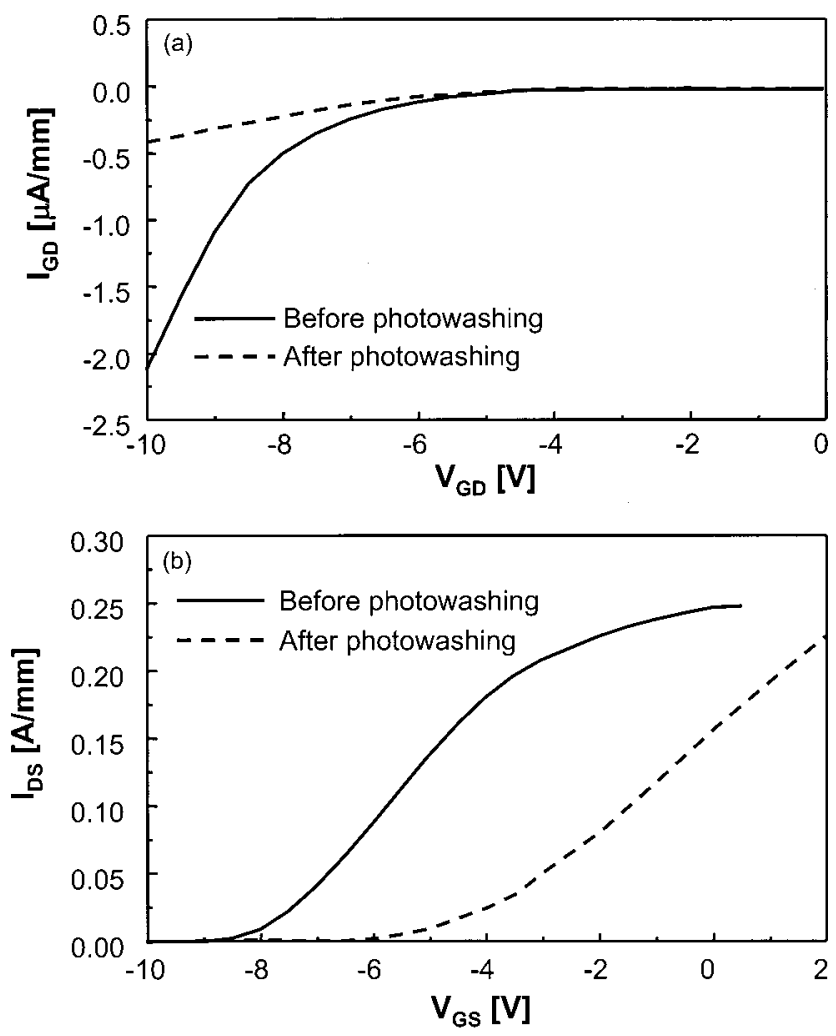

FIG. 1. Change in $I-V$ characteristics with photowashing treatment: (a) $I_{G D}$ vs $V_{G D}$ and (b) $I_{D S}$ vs $V_{G S}$.

The negative $G_{m}$ dispersion could be interpreted by electron trapping at the surface states on the ungated region between the gate and source/drain electrodes. ${ }^{8}$ In other words, during the negative half cycle of the sinusoidal signal, electrons are emitted from the gate to the ungated surface region. The electrons emitted can be captured by surface states on the ungated surface. At frequencies lower than the emission rate of an electron from the trap, electrons trapped at surface states are capable of following the sinusoidal signal applied.

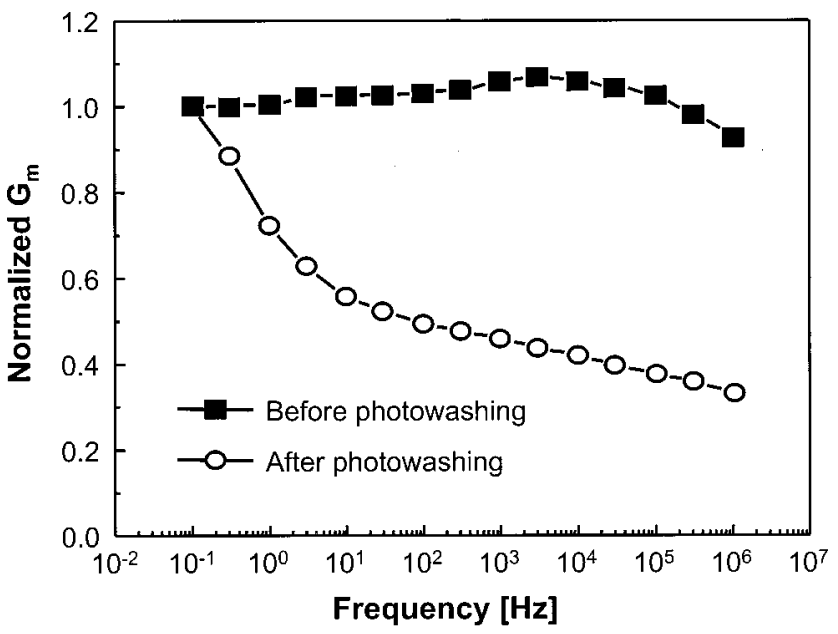

FIG. 2. Change in transconductance dispersion of the AlGaN/GaN HFET before and after photowashing treatment.

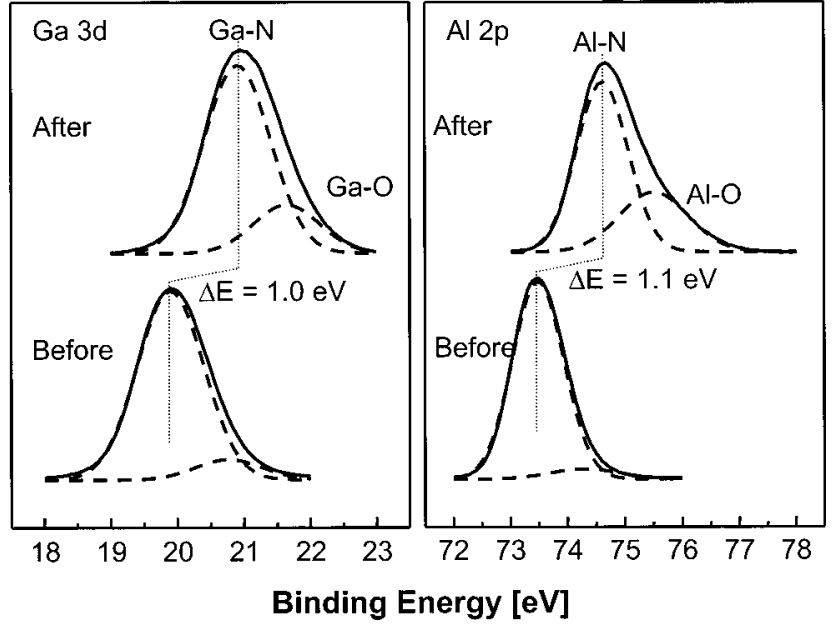

FIG. 3. Photoemission spectra of $\mathrm{Ga} 3 d$ and $\mathrm{Al} 2 p$ before and after a $10 \mathrm{~min}$ treatment at $\theta=90^{\circ}$.

Thus, the depletion region under the ungated surface as well as that under the gate is fully modulated by the signal applied. At higher frequencies, however, trapped electrons are unable to follow the signal and the charges held at surface states will be frozen. Thus, $G_{m}$ at higher frequencies becomes small, as shown in Fig. 2. From this, it is suggested that the photowashing treatment produced surface states at the ungated surface.

Figure 3 displays Ga $3 d$ and $\mathrm{Al} 2 p$ photoemission spectra at the surface of $\mathrm{AlGaN}$. The spectra before the treatment showed an asymmetric shape, which means another bond was superimposed on the peaks. Considering full widths at half maximum (FWHMs) and binding energies of chemical bonds, $\mathrm{Ga}-\mathrm{O}$ and $\mathrm{Al}-\mathrm{O}$ bonds could be separated from the Ga $3 d$ and $\mathrm{Al} 2 p$ spectra, respectively. After the treatment, the FWHM of each peak became broad, which is due to the increase of the oxide peaks. Note that even though the $\mathrm{Al}$ mole fraction in the AlGaN layer is only $25 \%$, the increase of the $\mathrm{Al}-\mathrm{O}$ bond was more significant than that of the $\mathrm{Ga}-\mathrm{O}$ bond. This can be explained using the Gibb's free energy change for the formation of each oxide. Namely, $-\Delta G_{298}$ in

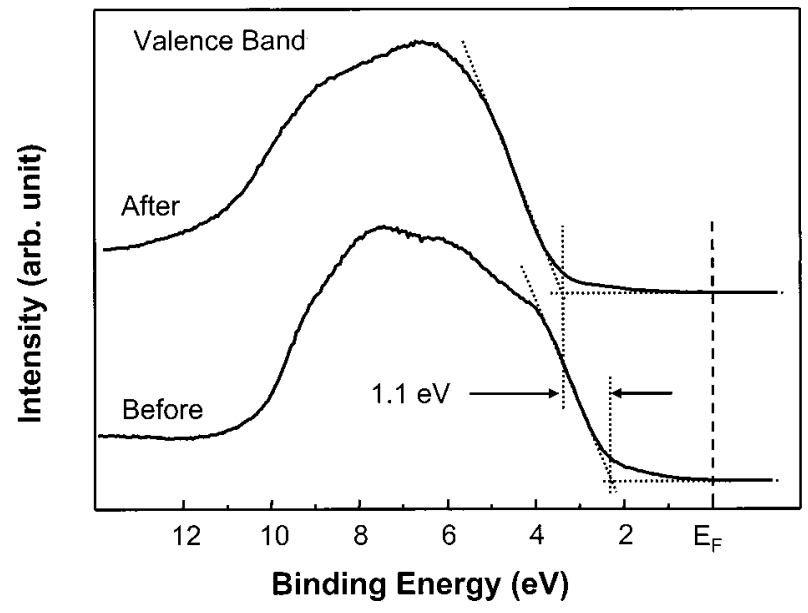

FIG. 4. Change in valence band spectra with photowashing treatment. 
TABLE I. Atomic concentration of each bond at the surface of AlGaN with $\theta$ and with the photowashing (PW) treatment.

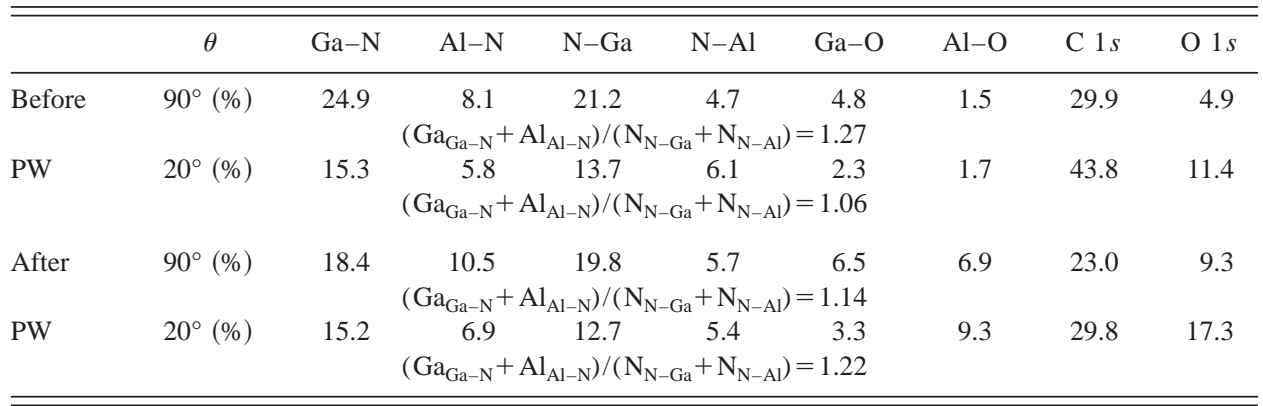

the reaction of $2 \mathrm{Al}+3 / 2 \mathrm{O}_{2} \rightarrow \mathrm{Al}_{2} \mathrm{O}_{3}$ is $1584 \mathrm{~kJ} / \mathrm{mol}$ larger than the $992 \mathrm{~kJ} / \mathrm{mol}$ of $2 \mathrm{Ga}+3 / 2 \mathrm{O}_{2} \rightarrow \mathrm{Ga}_{2} \mathrm{O}_{3}$.

The binding energies in $\mathrm{Ga}-\mathrm{N}$ and $\mathrm{Al}-\mathrm{N}$ bonds increased by $\sim 1.0 \mathrm{eV}$ as seen in Fig. 3. This means that the Fermi level at the surface of $\mathrm{AlGaN}$ was elevated by the same magnitude toward the conduction band edge. The increase in the Fermi level position by the treatment is directly confirmed by the valence band spectra in Fig. 4. The difference in energy between the valence band edge and Fermi level increased about $1.1 \mathrm{eV}$ after the treatment. This agrees well with the result in Fig. 3. The increase in the Fermi level means a reduction of band bending below the AlGaN surface.

In order to obtain depth information on atomic compositions at the surface of AlGaN, $\theta$ was varied. At a smaller take-off angle, the intensity of photoelectrons emitting from the surface becomes dominant due to the inelastic mean-free path of photoelectrons. The atomic concentrations of $\mathrm{Ga}-\mathrm{N}$, $\mathrm{Al}-\mathrm{N}, \mathrm{N}-\mathrm{Ga}$, and $\mathrm{N}-\mathrm{Al}$ bonds were determined using the peak area and the atomic sensitivity factor of each element, summarized in Table $\mathrm{I}$. The ratio of $\left(\mathrm{Al}_{\mathrm{Al}-\mathrm{N}}\right.$ $\left.+\mathrm{Ga}_{\mathrm{Ga}-\mathrm{N}}\right) /\left(\mathrm{N}_{\mathrm{N}-\mathrm{Ga}}+\mathrm{N}_{\mathrm{N}-\mathrm{Al}}\right)$ increased with the photowashing treatment at $\theta=20^{\circ}$. This means that the AlGaN surface became $\mathrm{N}$ deficient and a number of $\mathrm{N}$ vacancies ${ }^{9} V_{\mathrm{N}}$ were produced by the photowashing treatment.

The decreases in both $I_{G D}$ and $I_{D S}$ can be explained by the delay in the movement of electrons by trapping and detrapping mechanisms taking the results of $G_{m}$ dispersion and SRPES measurements into consideration. In the absence of surface states, electrons ejected into the bulk and/or the ungated surface under negative gate bias ${ }^{10}$ are swept directly into the drain. After the photowashing treatment, however, electrons ejected were captured by surface states and drifted toward the drain through the repetition of trapping and detrapping processes. This resulted in the decrease of $I_{G D}$ shown in Fig. 1(a). In addition, it is known that electrons trapped at surface states play a role in increasing the depletion layer width to maintain charge balance. ${ }^{8}$ This leads to the decrease in $I_{D S}$ via the reduction in the density of twodimensional electron gas (2DEG) at the interface of AlGaN with GaN, as shown in Fig. 1(b).

The decrease in the density of 2DEG may be attributed to the decrease in the thickness of AlGaN layer through the formation of the photowashing-induced oxide, because the $2 \mathrm{DEG}$ density increases with the thickness of $\mathrm{AlGaN}$ due to the effects of piezoelectric and spontaneous polarization. ${ }^{11}$ The oxide thickness can be estimated using the inelastic mean-free path of photoelectrons $\lambda$, defined as the escape probability of photoelectrons which decreases to 1/e with respect to the probability at the surface. The $\lambda$ of $\mathrm{Al} 2 p$ photoelectrons in $\mathrm{Al}_{2} \mathrm{O}_{3}$ was determined to be about $10 \AA$ using the relationship, $\lambda=2170 / E^{2}+0.72 a^{1.5} E^{0.5}$, where $E$ is the kinetic energy of $\mathrm{Al} 2 p$ photoelectrons and $a$ is the monolayer thickness of $\mathrm{Al}_{2} \mathrm{O}_{3} \cdot{ }^{12}$ The integral intensity of the $\mathrm{Al}-\mathrm{O}$ peak is about 0.47 in comparison with that of the Al-N peak, shown in Fig. 3, meaning that the Al-oxide thickness corresponds to $20 \AA \AA^{13}$ Thus the photowashinginduced oxide thickness is not sufficient to reduce $I_{D S}$, as shown in Fig. 1(b). From this, it is suggested that the photowashing treatment produced a number of $V_{\mathrm{N}}$ at the AlGaN surface, and that it plays a major role in reducing the $I_{D S}$ of HFETs.

In summary, both $I_{G D}$ and $I_{D S}$ simultaneously decreased after photowashing treatment on an AlGaN/GaN HFET. The treatment generated group-III oxide on the surface of AlGaN, leaving surface states behind in the ungated surface region, which was seen by the negative $G_{m}$ dispersion. The surface states generated by the photowashing treatment were suggested to be $\mathrm{N}$ vacancies, based upon the angle dependence of the atomic composition and the increase in binding energy in each element. The decrease of $I_{G D}$ was explained by a delay in the movement of electrons, namely, trapping and detrapping. The trapped electrons reduced the effective channel thickness, leading to the reduction of $I_{D S}$.

\section{ACKNOWLEDGMENT}

This work was performed through project for "National Research Laboratory" sponsored by the Korea Institute of Science and Technology Evaluation and Planning (KISTEP).

${ }^{1}$ V. Kumar, W. Lu, F. A. Khan, R. Schwindt, A. Kuliev, G. Simin, J. Yang, M. A. Khan, and I. Adesida, Electron. Lett. 38, 252 (2002).

${ }^{2}$ Y.-F. Wu, D. Kapolnek, J. P. Ibbetson, P. Parikh, B. P. Keller, and U. K. Mishra, IEEE Trans. Electron Devices ED-48, 586 (2001).

${ }^{3}$ E. D. Haberer, C.-H. Chen, A. Abare, M. Hansen, S. DenBaars, L. Coldren, U. Mishra, and E. L. Lu, Appl. Phys. Lett. 76, 3941 (2000).

${ }^{4}$ C.-H. Chen, S. Keller, E. D. Haberer, L. Zhang, S. P. DenBaars, E. L. Hy, and Y. Wu, J. Vac. Sci. Technol. B 17, 2755 (1999).

${ }^{5}$ H. Maher, D. W. Disanto, G. Soerensen, C. R. Bolognesi, H. Tand, and J. B. Webb, Appl. Phys. Lett. 77, 3833 (2000).

${ }^{6}$ C. Youtsey, I. Adesida, and G. Bulman, Appl. Phys. Lett. 71, 2151 (1997). 
${ }^{7}$ C. W. Wilmsen, P. D. Kirchner, J. M. Baker, D. T. Mclnturff, G. D. Pettit, and J. M. Woodall, J. Vac. Sci. Technol. B 6, 1180 (1988).

${ }^{8}$ K. J. Choi and J.-L. Lee, IEEE Trans. Electron Devices ED-48, 190 (2001).

${ }^{9}$ C. G. Van de Walle, Phys. Rev. B 56, 10020 (1997).

${ }^{10}$ T. M. Barton and P. H. Ladbrooke, Solid-State Electron. 29, 807 (1986).
${ }^{11}$ J. P. Ibbetson, P. T. Fini, K. D. Ness, S. P. DenBaars, J. S. Speck, and U. K. Mishra, Appl. Phys. Lett. 77, 250 (2000).

${ }^{12}$ Surface Analysis Methods in Materials Science, edited by D. J. O'Connor, B. A. Sexton, and R. C. Smart (Springer, Berlin, 1992), pp. 22-25.

${ }^{13}$ Handbook of X-ray and Ultraviolet Photoelectron Spectroscopy, edited by D. Griggs (Heyoen, London, 1977), pp. 158-159. 